\title{
Theory of Ultrafast Demagnetization after Femtosecond Laser Pulses
}

\author{
M. FÄhnle*, Ch. Illg, M. HaAG AND N. TEeny \\ Max Planck Institute for Intelligent Systems, Heisenbergstr. 3, 70569 Stuttgart, Germany
}

\begin{abstract}
Experimentally it has been found that a ferromagnetic metallic film is strongly demagnetized within few hundred fs after exposure to a fs laser pulse. The theories of this ultrafast demagnetization are reviewed, especially the influence of scatterings of the excited electrons at phonons and at magnons. The calculations for phonons and magnons are performed by the ab initio spin-density-functional electron theory for $\mathrm{Ni}$ and $\mathrm{Fe}$, and they are based on Fermi's golden rule for transition rates. The application of this rule on the fs time scale is critically discussed in view of results from quantum-kinetic density-matrix calculations. It is shown that the experimentally observed demagnetization rates cannot be explained by spin-flip scatterings of electrons exclusively at phonons or exclusively at magnons. A combination of individual spin-flip electron-phonon and spin-flip electron-magnon processes is shown to be a potential candidate for the explanation of ultrafast demagnetization.
\end{abstract}

DOI: 10.12693 /APhysPolA.127.170

PACS: 75.78.Jp, 75.30.Ds, 75.40.Gb

\section{Introduction}

The direction of the magnetization in a magnetic region may be interpreted as bit " 0 " for one direction and as bit "1" for the opposite direction. Therefore microand nanosized magnets can potentially be used in advanced data storage and data processing devices. It is desirable to increase the data storage density by miniaturization of the components, and to increase the speed of writing of the bits, i.e., it should be possible to modify the magnetization direction of the magnetic systems as fast as possible. Usually the magnetization direction in homogeneously magnetized films of lateral dimensions of micrometers and thicknesses of nanometers is switched by the application of magnetic fields or of spin-polarized currents. Another example is the switching of the polarization direction of a magnetic vortex core in a cylindrical disc film by small-amplitude rotating in-plane fields which hold for many periods or which are short bursts down to one period, or which are produced by short linear field pulses in two orthogonal in-plane directions with some time-delay between their respective starts. The minimum switching time which could be achieved so far was smaller than 100 ps [1].

Another way of changing the magnetization is the irradiation of the film with a fs optical laser pulse. In 1996, Beaurepaire et al. [2] showed for the first time that a thin ferromagnetic Ni film demagnetizes within few hundreds of femtoseconds (fs) after irradiation with a fs linearly polarized laser pulse. The demagnetization is followed by a remagnetization on a time scale which is much longer. Because of this remagnetization the effect of course cannot be used in information storage and data processing devices, but the physics is extremely interesting from

* corresponding author; e-mail: faehnle@is.mpg.de a fundamental point of view (see below). Later publications (for reviews, see Refs. [3, 4]) showed that by application of high-power fs lasers the time for demagnetization could be reduced to below 100 fs for Fe, Co and $\mathrm{Ni}$. This is presently considered as the fastest possible macroscopic manipulation of the magnetic state of a solid. Due to this fact it is called ultrafast magnetization dynamics. A fast demagnetization occurs not only for the $3 d$ transition metals $\mathrm{Fe}, \mathrm{Co}, \mathrm{Ni}$, but also for $4 f$ rare-earth metals, e.g., Gd, albeit in two steps, an initial reduction of the magnetization by about $25 \%$ in about $1 \mathrm{ps}$ and a final demagnetization in about $40 \mathrm{ps}$. The diversity of laser-induced demagnetization of type I systems (e.g., Fe, Co, Ni at not too high fluences) and type II systems (e.g., Gd) is explained in Ref. [3]. In the present paper we consider only type I systems.

Another fast magnetization process after short laser pulses is the all-optical switching. Irradiating ferrimagnetic films composed of rare-earth atoms and transitionmetal atoms (e.g., GdFeCo) with short laser pulses leads to a reproducible magnetization reversal in about several ps [5]. Because of the reproducibility of the magnetization reversal this effect potentially may be used for information storage and data processing devices. The mechanisms of ultrafast demagnetization and of alloptical switching are most probably different in detail. We will not consider all-optical switching.

In Sect. 2 the existing theories of ultrafast demagnetization are reviewed. Especially the basic assumption of all these theories, the conservation of the angular momentum of the sample and the involved photons is discussed critically. In Sect. 3 the calculational methods for the contributions of spin-flip scattering processes of the excited electrons at phonons and at magnons are described. Thereby, special emphasis is given on the questions which methods should be used to describe the processes on a fs time scale, Fermi's golden rule for transition rates or quantum-kinetic approaches. Section 4 gives the results. 
It will be shown that the experimentally observed demagnetization rates cannot be explained by spin-flip scatterings of electrons exclusively at phonons or exclusively at magnons. It will be discussed that possibly a combination of individual spin-flip electron-phonon and spinflip electron-magnon processes can describe the ultrafast demagnetization, as suggested by Schellekens and Koopmans [6]. Conclusions will be given in Sect. 5 .

\section{Review of theories of ultrafast demagnetization}

The magnetization $\boldsymbol{M}$ of a solid in the ground state is built by the expectation values of the electronic spin angular momentum $\langle\widehat{\boldsymbol{S}}\rangle$ and of the electronic orbital magnetic moment $\langle\widehat{\boldsymbol{L}}\rangle$,

$$
\boldsymbol{M}=\mu_{\mathrm{B}}\left(g_{\mathrm{s}}\langle\widehat{\boldsymbol{S}}\rangle+\langle\widehat{\boldsymbol{L}}\rangle\right),
$$

where $\mu_{\mathrm{B}}$ is Bohr's magneton and where $g_{\mathrm{s}}$ is the spin$g$-factor. In the following we consider films of bcc Fe and fcc Ni. In cubic transition metals $\langle\widehat{\boldsymbol{L}}\rangle$ is almost completely quenched by the crystal field, and therefore $\langle\widehat{\boldsymbol{S}}\rangle$ builds the magnetization to a good approximation. For the ultrafast demagnetization the question arises how the angular momentum can be transferred from the spin system of the film to other degrees of freedom on a $100 \mathrm{fs}$ time scale. Possible mechanisms will be discussed in the present section.

Battiato et al. [7] have argued that the diffusion of spin-polarized excited electrons from the film to a metallic substrate contributes to the demagnetization. However, ultrafast demagnetization is observed also for insulating substrates for which the effect of this "superdiffusion" is certainly small. We will neglect this effect in the following.

The basic assumption of all theories is that the total angular momentum $\langle\widehat{\boldsymbol{J}}\rangle$ of the film and the involved photons is conserved during the demagnetization, i.e.,

$$
\Delta\langle\widehat{\boldsymbol{J}}\rangle=\Delta\langle\widehat{\boldsymbol{S}}\rangle+\Delta\langle\widehat{\boldsymbol{L}}\rangle+\Delta\left\langle\widehat{\boldsymbol{L}}_{\mathrm{l}}\right\rangle+\Delta\left\langle\widehat{\boldsymbol{L}}_{\mathrm{ph}}\right\rangle=0,
$$

where $\left\langle\widehat{\boldsymbol{L}}_{\mathrm{l}}\right\rangle$ is the angular momentum of the lattice and $\left\langle\widehat{\boldsymbol{L}}_{\mathrm{ph}}\right\rangle$ is the angular momentum of the involved photons.

The conservation of $\langle\widehat{\boldsymbol{J}}\rangle$ is fulfilled if

1. the system under consideration is isotropic.

2. the Hamiltonian used in the theory includes all mutual interactions between the various degrees of freedom.

Both of these assumptions are not strictly fulfilled in the ultrafast demagnetization experiments. First, the system under consideration is not really isotropic, because the sample is not free in space but fixed on a sample holder which is fixed on a table which stands on the floor which is part of the earth. However, it is argued that on the fs time scale the transfer of angular momentum from the sample to the surroundings can be neglected [8]. Second, linearly polarized electromagnetic waves are emitted from the sample because its magnetization changes in time [9]. The waves emitted to the vacuum do not carry away angular momentum [8]. However, the linearly polarized waves may be represented as a superposition of right- and left-circularly polarized waves, and in a dichroic film the interaction of the emitted waves with the electrons is different for the two parts. Therefore, in a realistic non-spherical sample there is a net transfer of angular momentum from the waves to the sample by reabsorption. This is not taken into account in the existing theories (i.e., point 2. is not fulfilled), but the effect is small. So the effects of deviations from the strict conservation of angular momentum in the existing theories for ultrafast demagnetization in real samples are small and can be neglected [8].

In metallic films the dominant part of the demagnetization is observed after the duration of the laser pulse, so that the laser photons need not be considered as other degrees of freedom. So, altogether, the term $\Delta\left\langle\widehat{\boldsymbol{L}}_{\mathrm{ph}}\right\rangle$ in Eq. (2) can be neglected, and the interplay between $\Delta\langle\widehat{\boldsymbol{S}}\rangle, \Delta\langle\widehat{\boldsymbol{L}}\rangle$ and $\Delta\left\langle\widehat{\boldsymbol{L}}_{1}\right\rangle$ has to be considered. The most popular candidate for this interplay is a mechanism of Elliott-Yafet type $[10,11]$. The basic idea is that in a system with spin-orbit coupling the electronic states $\Psi_{j k}$ (with band index $j$ and wave vector $\boldsymbol{k}$ ) are no pure spin states, but spin-mixed states according to

$$
\Psi_{j \boldsymbol{k}}=\left[a_{j \boldsymbol{k}}(\boldsymbol{r})|\uparrow\rangle+b_{j \boldsymbol{k}}(\boldsymbol{r})|\downarrow\rangle\right] \exp (\mathrm{i} \boldsymbol{k} \boldsymbol{r}),
$$

where $a_{j \boldsymbol{k}}(\boldsymbol{r})$ and $b_{j \boldsymbol{k}}(\boldsymbol{r})$ are lattice periodic functions and $|\uparrow\rangle,|\downarrow\rangle$ are the two spinor eigenfunctions of $\widehat{S}_{z}$. The wave function is denoted as "dominant spin up" or "dominant spin down" if $p_{j \boldsymbol{k}}^{\uparrow}=\left\langle\Psi_{j \boldsymbol{k}} \mid \uparrow\right\rangle\left\langle\uparrow \mid \Psi_{j \boldsymbol{k}}\right\rangle$ is larger or less than $p_{j k}^{\downarrow}=\left\langle\Psi_{j k} \mid \downarrow\right\rangle\left\langle\downarrow \mid \Psi_{j k}\right\rangle$. In the following we denote the dominant spin character by $\widetilde{m}_{\mathrm{s}}$, and we add the index $\widetilde{m}_{\mathrm{s}}$ (which is determined by $j \boldsymbol{k}$ ) to the wave function, $\Psi_{j \boldsymbol{k} \widetilde{m}_{\mathrm{s}}}$. In such systems any scattering process between two states $\Psi_{j \boldsymbol{k} \widetilde{m}_{\mathrm{s}}}$ and $\Psi_{j^{\prime} \boldsymbol{k}^{\prime} \widetilde{m}_{\mathrm{s}}^{\prime}}$ changes the spin mixture, i.e., $\left\langle\Psi_{j \boldsymbol{k} \widetilde{m}_{\mathrm{s}}}\left|\widehat{S}_{z}\right| \Psi_{j \boldsymbol{k} \widetilde{m}_{\mathrm{s}}}\right\rangle$ is different from $\left\langle\Psi_{j^{\prime} \boldsymbol{k}^{\prime} \widetilde{m}_{\mathrm{s}}^{\prime}}\left|\widehat{S}_{z}\right| \Psi_{j^{\prime} \boldsymbol{k}^{\prime} \widetilde{m}_{\mathrm{s}}^{\prime}}\right\rangle$. With the transition rates $W_{j \boldsymbol{k} \widetilde{m}_{\mathrm{s}}, j^{\prime} \boldsymbol{k}^{\prime} \widetilde{m}_{\mathrm{s}}}$ for the transitions from $\Psi_{j \boldsymbol{k} \widetilde{m}_{\mathrm{s}}}$ to $\Psi_{j^{\prime} \boldsymbol{k}^{\prime} \widetilde{m}_{\mathrm{s}}}$ the rate of change of the magnetic moment per atom can be calculated when modeling the occupation numbers of the electronic states directly after the laser pulse (for details see Sect. 3). The transition rates depend on the scattering operator which is different for different scattering mechanisms. Examples are electron-phonon scatterings, electron-magnon scatterings, electron-electron scatterings and electron-defect or electron-interface scatterings. The latter types of scatterings so far have not been considered for the ultrafast demagnetization, because it was found experimentally that the demagnetization is very similar for samples with different structural properties (epitaxial films, polycrystalline films, etc.). Many papers are based on electron-phonon scatterings (see, e.g., Ref. [12] where the electron-phonon scattering is treated by the ab initio electron theory). The contributions of electron-magnon scatterings are discussed in Refs. [6, 13] and are treated by the ab initio theory in Ref. [14]. 
References $[15,16]$ consider with non-ab initio model calculations the contributions of electron-electron scatterings. It should be noted that in all theories only the change of the expectation value of the magnetic moment is calculated which is related to a change of the angular momentum of the magnetic species (electrons, magnons). However, the theories do not keep track of the angular momentum transfer from the magnetic species to the lattice, they assume implicitly that the lattice is a perfect sink for the angular momentum. In Sect. 4 we will discuss our results for the contributions of the electronphonon and the electron-magnon scattering. It will be shown that the experimentally observed demagnetization rates of $\mathrm{Ni}$ and $\mathrm{Fe}$ cannot be explained by scattering of the excited electrons exclusively at phonons or exclusively at magnons, but possibly by a combination of individual electron-phonon and electron-magnon processes. In Refs. [15, 16] it is concluded from model calculations that the electron-electron scatterings dominate the ultrafast demagnetization. We think that this has to be checked by corresponding ab initio calculations.

Finally, it has been argued in Ref. [12] that on the time scale of fs the phonon-phonon interactions and the phonon-magnon interactions can be neglected, and the same holds for magnon-magnon interactions.

\section{Computational methods}

In this section we summarize the basic assumptions which we make in our calculations of the contributions of electron-phonon and electron-magnon scatterings to the ultrafast demagnetization after fs laser pulses. For details of the calculational methods we refer to Refs. [12, 14].

A complete analysis of the demagnetization behaviour would require the solution of the time-dependent manybody Schrödinger equation for electrons and atoms, where the spin-orbit coupling and the interaction of the system with the laser pulse has to be taken into account. From the obtained electron magnetization density $\boldsymbol{m}(\boldsymbol{r}, t)$ the atomic magnetic moments $\boldsymbol{M}_{\boldsymbol{R}}(t)=$ $M_{\boldsymbol{R}}(t) \cdot \boldsymbol{e}_{\boldsymbol{R}}(t)$ with magnitude $M_{\boldsymbol{R}}(t)$ and orientation $\boldsymbol{e}_{\boldsymbol{R}}(t)$, defined by

$$
\boldsymbol{M}_{\boldsymbol{R}}(t)=\frac{\nu}{2} \int_{\Omega_{\boldsymbol{R}}} \mathrm{d}^{3} r^{\prime} \int_{t-\frac{1}{\nu}}^{t+\frac{1}{\nu}} \mathrm{d} t^{\prime} \boldsymbol{m}\left(\boldsymbol{r}^{\prime}, t^{\prime}\right),
$$

can be calculated. Here $\Omega_{\boldsymbol{R}}$ is the volume of the atomic sphere around the nucleus at position $\boldsymbol{R}$, and $\nu$ is the frequency, $\nu \approx 10^{13} \mathrm{~Hz}$, of a typical long-wavelength magnon. In a first step of approximation, the full Hamiltonian is replaced by a model Hamiltonian

$$
\begin{aligned}
\widehat{H} & =\widehat{H}_{\text {electron }}+\widehat{H}_{\text {phonon }}+\widehat{H}_{\text {magnon }} \\
& +\widehat{H}_{\text {electron-phonon }}+\widehat{H}_{\text {electron-magnon }},
\end{aligned}
$$

which describes non-interacting electrons (in the sense of the Kohn-Sham density functional theory), noninteracting phonons, non-interacting magnons, electronphonon interactions and electron-magnon interactions. Because we do not consider electron-electron scatterings, we omit the Coulomb-interaction term between electrons, and on the fs time scale we also can omit terms describing phonon-phonon, magnon-magnon and phonon-magnon interactions (see above). Finally, we omit a term describing the interaction of the laser beam with the film and model (see Sect. 3.1) instead the state of the system directly after the laser beam by appropriate occupation numbers for electrons, phonons and magnons.

\subsection{Elliott-Yafet model of the driving force for demagnetization}

Directly after the laser pulse the temperatures of the phonon and of the magnon system are equal to the temperature at which the experiment is performed (we use room temperature), and the occupation numbers are given by corresponding Bose distribution functions. For the electrons, the situation is more complicated. We model their non-equilibrium occupation numbers by the Elliott-Yafet scenario (Fig. 3 of Ref. [4]) with two assumptions. First, the laser beam excites the electrons. Electron-electron scatterings lead very rapidly to a thermalization of the electronic system, yielding an electron temperature $T_{\mathrm{el}}$ which is - depending on the laser fluence - typically between $500 \mathrm{~K}$ and $900 \mathrm{~K}[3,17,18]$. As discussed in Ref. [3], the demagnetization is dominated by processes after this very rapid thermalization. However, the temperature difference between electrons and phonons/magnons is not the only driving force for demagnetization. An additional driving force is a difference between the chemical potentials $\varepsilon_{\mathrm{F}}\left(\widetilde{m}_{\mathrm{S}}\right)$ for states with different $\widetilde{m}_{\mathrm{s}}$, and the corresponding occupation numbers for the electronic states $\Psi_{j \boldsymbol{k}} \widetilde{m}_{\mathrm{s}}$ are given by the Fermi distribution $f\left(\varepsilon_{j \boldsymbol{k}} \widetilde{m}_{\mathrm{s}}, \varepsilon_{\mathrm{F}}\left(\widetilde{m}_{\mathrm{S}}\right), T_{\mathrm{el}}\right)$. The $\varepsilon_{\mathrm{F}}\left(\widetilde{m}_{\mathrm{s}}\right)$ directly after the laser pulse are calculated from the demands that the electronic magnetic moment per atom still has the value $M_{0}$ as before the pulse and that an integral of $f$ over all states $(j \boldsymbol{k})$ gives the total number $N_{0}$ of valence electrons per atom.

\subsection{Scattering of non-equilibrium electrons at phonons and magnons}

The quasiparticles (phonons, magnons) represent timeperiodic perturbations of the electronic states of the unperturbed system leading to transitions between various states, i.e., electrons are scattered at the quasiparticles. Our basic assumption is that the development of the system in time may be described by many incoherent instantaneous scattering processes between single electrons and single quasiparticles which do not depend on preceding scattering processes (the Boltzmann type approach). This Markovian property is reproduced in a first-order time-dependent perturbation theory. The higher orders can be neglected if the perturbation time is short and if the perturbation strength is weak. If the perturbation time is much longer than $2 \pi \hbar / \Delta \varepsilon$ where $\Delta \varepsilon$ is the energy difference between the initial and the final electron states

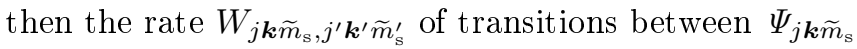
and $\Psi_{j^{\prime} \boldsymbol{k}^{\prime} \widetilde{m}_{\mathrm{s}}^{\prime}}$ is described by Fermi's golden rule,

$$
W_{j \boldsymbol{k} \widetilde{m}_{\mathrm{s}}, j^{\prime} \boldsymbol{k}^{\prime} \widetilde{m}_{\mathrm{s}}^{\prime}}^{\mathrm{A}, \mathrm{E}}=\frac{2 \pi}{\hbar} \mid M_{j \boldsymbol{k} \widetilde{m}_{\mathrm{s}}, j^{\prime} \boldsymbol{k}^{\prime} \widetilde{m}_{\mathrm{s}}^{\prime}}^{\mathrm{A},\left.\right|^{2}}
$$




$$
\times \delta\left(\varepsilon_{j^{\prime} \boldsymbol{k}^{\prime} \widetilde{m}_{\mathrm{s}}^{\prime}}-\left(\varepsilon_{j \boldsymbol{k} \widetilde{m}_{\mathrm{s}}} \pm \hbar \omega_{\text {qquasi }}\right)\right),
$$

where $\boldsymbol{q}_{\text {quasi }}= \pm\left(\boldsymbol{k}^{\prime}-\boldsymbol{k}+\boldsymbol{G}\right)$ with a reciprocal lattice vector $\boldsymbol{G}$ which has to be added if $\boldsymbol{k}^{\prime}-\boldsymbol{k}$ is outside the first Brillouin zone, and the $+(-)$ sign holds for the absorption (A) or the emission (E) of the quasiparticle. The quantities $M_{j \boldsymbol{k}}^{\mathrm{A}, \mathrm{E}} \tilde{m}_{\mathrm{s}, j^{\prime} \boldsymbol{k}^{\prime} \tilde{m}_{\mathrm{s}}^{\prime}}$ are the transition matrix elements (see Sect. 3.3). Inserting for $\Delta \varepsilon$ a large quasiparticle energy, e.g., $40 \mathrm{meV}$ for phonons in $\mathrm{Ni}$, the perturbation time should be much larger than $100 \mathrm{fs}$ (which is in contrast to the demand of a short perturbation time to guarantee that the Markovian first-order theory holds). Since the ultrafast demagnetization is on a time scale of $100 \mathrm{fs}$, it is in principle not allowed to use Fermi's golden rule with the $\delta$-function which represents conservation of energy in the scattering process. In fact, $100 \mathrm{fs}$ is shorter than an oscillation period of typical phonons and magnons, so that the electron does not "know" the frequency of the quasiparticle, or - expressed in terms of the time-energy uncertainty relation - the energy is not well defined whereas Eq. (6) implies energy conservation. Furthermore, during $100 \mathrm{fs}$ an electron-quasiparticle scattering process is not yet completed, and therefore the various scattering processes are not "immediate" and Markovian. Altogether, this means that in principle the procedure based on Fermi's golden rule should be replaced by a quantum-kinetic calculation within the density-matrix approach which does not suffer from the above discussed problems. In this context we want to mention Ref. [19], where for pulse-excited semiconductors the transfer of energy between excited electrons and phonons has been investigated by a quantum-kinetic approach and by a Boltzmann type approach. The quantum-kinetic effects turned out to be very important when looking at spectral distribution functions. However, the results of the two calculations are already very similar $10 \mathrm{fs}$ after the laser pulse when looking at the time dependence of the average electron energy. The hope therefore is that the Boltzmann type Elliott-Yafet theory will also yield good results for the average spin magnetic moment which determines the demagnetization.

\subsection{Electron-phonon and electron-magnon scattering operators}

The transition matrix elements appearing in Eq. (6) are

$$
M_{j \boldsymbol{k} \widetilde{m}_{\mathrm{s}}, j^{\prime} \boldsymbol{k}^{\prime} \widetilde{m}_{\mathrm{s}}^{\prime}}^{\mathrm{A}}=\left\langle F^{\prime} \Psi_{j^{\prime} \boldsymbol{k}^{\prime} \widetilde{m}_{\mathrm{s}}^{\prime}}\left|W_{\boldsymbol{q}}^{\text {quasi; } \mathrm{A}, \mathrm{E}}\right| F \Psi_{j \boldsymbol{k} \widetilde{m}_{\mathrm{s}}}\right\rangle,
$$

where $|F\rangle$ and $\left|F^{\prime}\right\rangle$ characterize the quasiparticle state of the system (described by quasiparticle occupation numbers) before and after the scattering process. Thereby the scattering operator $W_{\boldsymbol{q}}$ quasi; A, E is a $(2 \times 2)$ matrix acting on the two-component spinor fields $\Psi_{j k \tilde{m}_{s}}$. For the case of phonons it describes the distortion of the electrostatic lattice potential and a distortion of the spin-orbit coupling, and it is given by Eq. (6) of Ref. [12]. The operator for the scattering of crystal electrons at magnons has been derived in Ref. [20] and is given by Eqs. (7)-(10) of Ref. [14]. The spinor fields
$\Psi_{j k \widetilde{m}_{\mathrm{s}}}$ occurring in the matrix elements are calculated by the linear-muffin-tin orbital (LMTO) theory [21] in atomic-sphere approximation (ASA), see Eq. (11) of Ref. [14].

\subsection{Calculation of the demagnetization rate and the demagnetization time}

Within the Elliott-Yafet Boltzmann type approach the rate of change of the magnetic moment per atom directly after the laser pulse is given by

$$
\begin{aligned}
& \Delta M=\frac{\mathrm{d} M}{\mathrm{~d} t}=\frac{1}{\Omega_{\mathrm{BZ}}^{2}} \sum_{j j^{\prime}} \int_{\mathrm{BZ}} \mathrm{d}^{3} k \int_{\mathrm{BZ}} \mathrm{d}^{3} k^{\prime} \Delta M_{j \boldsymbol{k} \widetilde{m}_{\mathrm{s}}, j^{\prime} \boldsymbol{k}^{\prime} \widetilde{m}_{\mathrm{s}}^{\prime}} \\
& \times\left\{f\left(\varepsilon_{j \boldsymbol{k} \tilde{m}_{\mathrm{s}}}, \varepsilon_{\mathrm{F}}\left(\widetilde{m}_{\mathrm{s}}\right), T_{\mathrm{el}}\right)\left[1-f\left(\varepsilon_{j^{\prime} \boldsymbol{k}^{\prime} \widetilde{m}_{\mathrm{s}}^{\prime}}, \varepsilon_{\mathrm{F}}\left(\widetilde{m}_{\mathrm{s}}^{\prime}\right), T_{\mathrm{el}}\right)\right]\right. \\
& \times W_{j \boldsymbol{k} \widetilde{m}_{\mathrm{s}}, j^{\prime} \boldsymbol{k}^{\prime} \tilde{m}_{\mathrm{s}}^{\prime}} \\
& -f\left(\varepsilon_{j^{\prime} \boldsymbol{k}^{\prime} \widetilde{m}_{\mathrm{s}}^{\prime}}, \varepsilon_{\mathrm{F}}\left(\widetilde{m}_{\mathrm{s}}^{\prime}\right), T_{\mathrm{el}}\right)\left[1-f\left(\varepsilon_{j \boldsymbol{k} \widetilde{m}_{\mathrm{s}}}, \varepsilon_{\mathrm{F}}\left(\widetilde{m}_{\mathrm{s}}\right), T_{\mathrm{el}}\right)\right] \\
& \left.\times W_{j^{\prime} \boldsymbol{k}^{\prime} \widetilde{m}_{\mathrm{s}}^{\prime}, j \boldsymbol{k} \widetilde{m}_{\mathrm{s}}}\right\},
\end{aligned}
$$

where $\Omega_{\mathrm{BZ}}$ is the volume of the Brillouin zone. $\Delta M_{j \boldsymbol{k} \tilde{m}_{s}, j^{\prime} \boldsymbol{k}^{\prime} \tilde{m}_{\mathrm{s}}^{\prime}}$ keeps track of the magnetic moment change for each electronic transition. For the case of electron-phonon scatterings it is approximated by the change $\Delta M_{j \boldsymbol{k} \widetilde{m}_{\mathrm{s}}, j^{\prime} \boldsymbol{k}^{\prime} \widetilde{m}_{\mathrm{s}}^{\prime}}^{\text {electron }}$ of the electronic magnetic moment. For the electron-magnon scattering we use

$$
\begin{gathered}
\Delta M_{j \boldsymbol{k} \widetilde{m}_{\mathrm{s}}, j^{\prime} \boldsymbol{k}^{\prime} \widetilde{m}_{\mathrm{s}}^{\prime}}=\Delta M_{j \boldsymbol{k} \widetilde{m}_{\mathrm{s}}, j^{\prime} \boldsymbol{k}^{\prime} \widetilde{m}_{\mathrm{s}}^{\prime}}^{\text {electron,sin }}+\Delta M_{j \boldsymbol{k} \widetilde{m}_{\mathrm{s}}, j^{\prime} \boldsymbol{k}^{\prime} \widetilde{m}_{\mathrm{s}}^{\prime}}^{\text {electron orbital }} \\
\mp g \mu_{\mathrm{B}},
\end{gathered}
$$

where $\Delta M_{j \boldsymbol{k} \tilde{m}_{\mathrm{s}}, j^{\prime} \boldsymbol{k}^{\prime} \tilde{m}_{\mathrm{s}}^{\prime}}^{\text {electal }}$ is the change of the electronic magnetic orbital moment, and where the plus (minus) sign holds for magnon absorption (emission). Please note that in a system with spin-orbit coupling the absorption or the emission of a magnon does not change the magnetic moment exactly (but only in very good approximation) by $-g \mu_{\mathrm{B}}$ or $+g \mu_{\mathrm{B}}$.

If the decrease of the magnetization is of the form

$$
\frac{\mathrm{d} M}{\mathrm{~d} t}=-\frac{M(t)-M(t=0)}{T_{1}},
$$

then the demagnetization time $T_{1}$ is given by Eq. (15) of Ref. [12], which contains again the transition rates $W_{j \boldsymbol{k} \widetilde{m}_{\mathrm{s}}, j^{\prime} \boldsymbol{k}^{\prime} \tilde{m}_{\mathrm{s}}^{\prime}}$, but otherwise only equilibrium quantities, namely the chemical potential $\varepsilon_{\mathrm{F}}$ which in equilibrium is the same for dominant spin-up and for dominant spindown states, and the equilibrium temperature $T_{0}$ for which we insert room temperature (see Ref. [22]).

\section{Results \\ 4.1. Electron-phonon scatterings}

In this section we summarize the results for the contributions of electron-phonon scatterings to the ultrafast demagnetization which are discussed in detail in Ref. [12]. The calculated demagnetization time is $16 \mathrm{fs}$ for fcc Ni and 22 fs for bcc Fe. These times are smaller than experimental demagnetization times (about $100 \mathrm{fs}$ or $200 \mathrm{fs}$ ). This shows that electron-phonon scattering is fast enough to explain in principle a magnetization dynamics on a sub-100 fs time scale, in contrast 
to a wide-spread opinion that electron-phonon scatterings can change observables only on a time scale which is longer than the oscillation time of one phonon period which is typically $1 \mathrm{ps}$. However, the demagnetization rate $\mathrm{d} M / \mathrm{d} t$ which characterizes the strength of the demagnetization and which we calculated for electron temperatures up to $2000 \mathrm{~K}$ from Eq. (8) - whereby the typical values for $T_{\mathrm{el}}$ are between $500 \mathrm{~K}$ and $900 \mathrm{~K}$ as described in Sect. 3.1 - is very much smaller than the experimentally observed demagnetization for bcc Fe for all values of $T_{\mathrm{el}}$. For fcc $\mathrm{Ni}$ the calculated rates are also much smaller than the observed rates up to $T_{\mathrm{el}}=1000 \mathrm{~K}$ and only for unrealistic values $T_{\mathrm{el}} \geq 1500 \mathrm{~K}$ they are comparable to the experimental rates.

\subsection{Electron-magnon scatterings}

In a system without spin-orbit coupling electronmagnon scatterings conserve the spin angular momentum, i.e., they do not contribute to demagnetization. Assume, e.g., that a spin-down electron flips its spin orientation (leading to an increase of the magnetic moment by $2 \mu_{\mathrm{B}}$ ) by emission of a magnon, which - in turn leads to a decrease of the magnetic moment by $-2 \mu_{\mathrm{B}}$, so that - altogether - the magnetic spin moment is conserved. In a system with spin-orbit coupling the electronic states are no pure spin states (see Eq. (3)), and there is in addition a small expectation value $\langle\widehat{L}\rangle$ of the orbital angular momentum of these states which is related to an orbital magnetic moment (with a $g$-factor of 1 instead of $g_{\mathrm{s}}=2$ ). The absorption or emission of a magnon changes the magnetic moment of the system with spin orbit coupling only approximately (but not exactly) by 2 Bohr magnetons or -2 Bohr magnetons. The change of the total magnetic moment of the system per atom and per time due to electron-magnon scattering processes is

$$
\begin{gathered}
\Delta M=\Delta M_{\text {electron }}^{\text {spin }}+\Delta M_{\text {electron }}^{\text {orbital }} \\
+\Delta M_{\text {magnon }}^{\mathrm{A}}+\Delta M_{\text {magnon }}^{\mathrm{E}}
\end{gathered}
$$

If we assume with Carpene et al. that $\Delta M_{\text {electron }}^{\text {orbital im- }}$ mediately quenched by the crystal field, then the change of the total atomic magnetic moment is not $\Delta M$ given by Eq. (11) but

$$
\Delta M_{\text {Carpene }}=\Delta M-\Delta M_{\text {electron }}^{\text {orbital }} \text {. }
$$

Tables I and II represent our results for three different values of $T_{\mathrm{el}}$ for $\mathrm{Ni}$ and $\mathrm{Fe} . \Delta M_{\text {Carpene }}$ is negative both for $\mathrm{Ni}$ and $\mathrm{Fe}$, but the magnitudes are much smaller than the experimentally observed rates which are - depending on the laser fluence (which determines $T_{\mathrm{el}}$ ) - of the order of $0.5 \mu_{\mathrm{B}} / 100 \mathrm{fs}$. This means that the mechanism of Carpene et al. cannot explain the ultrafast demagnetization. $\Delta M_{\text {magnon }}^{\mathrm{E}}$ is much larger than $\Delta M_{\text {magnon }}^{\mathrm{A}}$.

\subsection{Discussion of the mechanism of Schellekens and Koopmans}

In Sects. 4.1 and 4.2 it has been shown that the experimentally observed demagnetization rates cannot be explained by scattering of electrons exclusively at

\section{TABLE I}

The various contributions to the total change $\Delta M$ of the atomic magnetic moment (in units of $\mu_{\mathrm{B}}$ ) per $100 \mathrm{fs}$ in $\mathrm{Ni}$. $T_{\mathrm{el}}$ is in units of K.

\begin{tabular}{c|r|r|r}
\hline \hline$T_{\text {el }}$ & \multicolumn{1}{|c|}{500} & \multicolumn{1}{c}{700} & \multicolumn{1}{c}{900} \\
\hline$\Delta M$ & $-2.10 \times 10^{-4}$ & $-5.09 \times 10^{-4}$ & $-9.92 \times 10^{-4}$ \\
$\Delta M_{\text {electron }}^{\text {orbital }}$ & $2.65 \times 10^{-3}$ & $8.48 \times 10^{-3}$ & $2.14 \times 10^{-2}$ \\
$\Delta M_{\text {magnon }}^{\mathrm{A}}$ & $2.21 \times 10^{-2}$ & $2.62 \times 10^{-2}$ & $3.04 \times 10^{-2}$ \\
$\Delta M_{\text {magnon }}^{\mathrm{E}}$ & $-5.66 \times 10^{-2}$ & $-1.36 \times 10^{-1}$ & $-3.08 \times 10^{-1}$ \\
$\Delta M_{\text {Carpene }}$ & $-2.86 \times 10^{-3}$ & $-8.99 \times 10^{-3}$ & $-2.24 \times 10^{-2}$
\end{tabular}

TABLE II

The various contributions to the total change $\Delta M$ of the atomic magnetic moment (in units of $\mu_{\mathrm{B}}$ ) per $100 \mathrm{fs}$ in Fe. $T_{\mathrm{el}}$ is in units of $\mathrm{K}$.

\begin{tabular}{c|c|c|c}
\hline \hline$T_{\text {el }}$ & 500 & \multicolumn{1}{|c|}{700} & 900 \\
\hline$\Delta M$ & $-3.22 \times 10^{-2}$ & $-7.86 \times 10^{-2}$ & $-1.40 \times 10^{-1}$ \\
$\Delta M_{\text {electron }}^{\text {orrital }}$ & $-1.06 \times 10^{-3}$ & $-2.10 \times 10^{-3}$ & $-8.64 \times 10^{-4}$ \\
$\Delta M_{\text {magnon }}^{\mathrm{A}}$ & $1.50 \times 10^{-1}$ & $1.65 \times 10^{-1}$ & $1.81 \times 10^{-1}$ \\
$\Delta M_{\text {magnon }}^{\mathrm{E}}$ & $-6.55 \times 10^{-1}$ & -1.49 & -2.69 \\
$\Delta M_{\text {Carpene }}$ & $-3.11 \times 10^{-2}$ & $-7.65 \times 10^{-2}$ & $-1.39 \times 10^{-1}$
\end{tabular}

phonons or exclusively at magnons. Schellekens and Koopmans [6] have discussed by simple model calculations that a stronger demagnetization can be produced when electron spin flips and atomic moment flips are respected. In Refs. [12] and [14] a microscopic interpretation of this model is given in terms of a combination of individual spin-flip electron-phonon scatterings and spin-flip electron-magnon scatterings. Apart from other mechanisms which - however - contribute very little, there are two dominant processes

$$
\begin{array}{rlrl}
\text { or } \quad & \text { phonon } \rightarrow \downarrow, & \downarrow \rightarrow \uparrow+\text { magnon, } \\
\uparrow \rightarrow \downarrow+\text { phonon, } & \downarrow \rightarrow \uparrow+\text { magnon. }
\end{array}
$$

Thereby, the electron-phonon scatterings deliver angular momentum from the spin system to the lattice, whereas in the respective magnon-emission process the original spin state of the electron is restored and the magnon disorders the atomic magnetic moments, leading to a demagnetization.

The idea behind this model is the following. It has been shown in Ref. [12] that the calculated demagnetization rate due to electron-phonon scatterings are smaller than the experimentally observed rates because the available phase space for the electron-phonon scattering is too small. The combination of electron-phonon and electron-magnon processes described by Eqs. (13), (14) leads to a demagnetization without consuming phase space for spin-flip electron-phonon scatterings (because at the end of the combined processes the original spin state of the electron is restored). In Ref. [14] a way is described to calculate explicitly the demagnetization by this combination of two scattering mechanisms, which - however - is extremely complicated and time- 
consuming. Therefore, so far only the necessary precondition for the validity of the model of Schellekens and Koopmans has been studied. This is that the rate $W_{\text {magnon }}^{\downarrow \uparrow}$ of scatterings from dominant spin-down to dominant spin-up electronic states by magnons should be at least of the same order as the rate $W_{\text {phonon }}^{\uparrow \downarrow}$ of scatterings from dominant spin-up to dominant spin-down states by phonons which can be calculated from the quantities given by Eq. (6). Then the total demagnetization rate would be determined by the electronphonon scattering processes. Because in the processes described by Eqs. (13), (14) no phase space for spinflip electron-phonon scatterings is consumed the demagnetization rates are larger than those for exclusively electron-phonon scatterings which consume phase space, i.e., larger than the demagnetization rates calculated in Sec. 4.1. Then possibly the calculated demagnetization rates for the combined processes are similar to the experimentally observed rates.

Tables III and IV give the results for $W_{\text {magnon }}^{\downarrow \uparrow}$ and $W_{\text {phonon }}^{\uparrow \downarrow}$ for various $T_{\text {el }}$ for $\mathrm{Ni}$ and Fe. Especially for $\mathrm{Fe}$ but also for $\mathrm{Ni}$ the above discussed precondition is fulfilled. Thus the mechanism of Schellekens and Koopmans is a candidate for the explanation of the experimentally observed ultrafast demagnetization.

TABLE III

Rates of scatterings of electrons at magnons and at phonons between different spin states, within 100 fs and per atom, in $\mathrm{Ni}$.

\begin{tabular}{c|l|l|l}
\hline \hline$T_{\mathrm{el}}$ & \multicolumn{1}{|c|}{500} & \multicolumn{1}{c}{700} & \multicolumn{1}{c}{900} \\
\hline$W_{\text {magnon }}^{\downarrow \uparrow}$ & $2.22 \times 10^{-2}$ & $5.20 \times 10^{-2}$ & $1.15 \times 10^{-1}$ \\
$W_{\text {phonon }}^{\uparrow \downarrow}$ & $4 \times 10^{-2}$ & $6 \times 10^{-2}$ & $8 \times 10^{-2}$
\end{tabular}

TABLE IV

Rates of scatterings of electrons at magnons and at phonons between different spin states, within $100 \mathrm{fs}$ and per atom, in Fe.

\begin{tabular}{c|c|l|l}
\hline \hline$T_{\text {el }}$ & \multicolumn{1}{|c|}{500} & \multicolumn{1}{c}{700} & \multicolumn{1}{c}{900} \\
\hline$W_{\text {magnon }}^{\downarrow \uparrow}$ & $3.16 \times 10^{-1}$ & $7.23 \times 10^{-1}$ & 1.30 \\
$W_{\text {phonon }}^{\uparrow \downarrow}$ & $3.50 \times 10^{-2}$ & $5 \times 10^{-2}$ & $6.5 \times 10^{-2}$
\end{tabular}

\section{Conclusions}

The experimentally observed demagnetization rates of transition-metal films after femtosecond laser irradiation cannot be explained by scattering of the excited electrons exclusively at phonons or exclusively at magnons. A potential candidate for the explanation of the experimental results is a combination of individual spin-flip electronphonon and spin-flip electron-magnon processes. It may also be that electron-electron scatterings may explain the ultrafast demagnetization in systems with spin-orbit coupling. This has been predicted in Refs. [15, 16] by model calculations, but this prediction should be checked by corresponding ab initio calculations.

\section{Acknowledgments}

The authors thank E. Carpene, B. Koopmans and A.J. Schellekens for helpful discussions.

\section{References}

[1] M. Noske, A. Gangwar, H. Stoll, M. Kammerer, M. Sproll, G. Dieterle, M. Weigand, M. Fähnle, G. Woltersdorf, C.H. Back, G. Schütz, Phys. Rev. $B$ 90, 104415 (2014).

[2] E. Beaurepaire, J.-C. Merle, A. Daunois, J.-Y. Bigot, Phys. Rev. Lett. 76, 4250 (1996).

[3] B. Koopmans, G. Malinowski, F. Dalla Longa, D. Steiauf, M. Fähnle, T. Roth, M. Cinchetti, M. Aeschlimann, Nat. Mater. 9, 259 (2010).

[4] M. Fähnle, C. Illg, J. Phys. Condens. Matter 23, 493201 (2011).

[5] I. Radu, K. Vahaplar, C. Stamm, T. Kachel, N. Pontius, H.A. Dürr, T.A. Ostler, J. Barker, R.F.L. Evans, R.W. Chantrell, A. Tsukamoto, A. Itoh, A. Kirilyuk, T. Rasing, A.V. Kimel, Nature 472, 205 (2011).

[6] A.J. Schellekens, B. Koopmans, Phys. Rev. Lett. 110, 217204 (2013).

[7] M. Battiato, K. Carva, P.M. Oppeneer, Phys. Rev. Lett. 105, 027203 (2010).

[8] M. Fähnle, M. Haag, C. Illg, J. Magn. Magn. Mater. 347, 45 (2013).

[9] E. Beaurepaire, G.M. Turner, S.M. Harrel, M.C. Beard, J.-Y. Bigot, C.A. Schmuttenmaer, Appl. Phys. Lett. 84, 3465 (2004).

[10] R.J. Elliott, Phys. Rev. 96, 266 (1954).

[11] Y. Yafet, in: Solid State Physics, Vol. 14, Eds. F. Seitz, D. Turnbull, Academic, New York 1963.

[12] C. Illg, M. Haag, M. Fähnle, Phys. Rev. B 88, 214404 (2013).

[13] E. Carpene, E. Mancini, C. Dallera, M. Brenna, E. Puppin, S. De Silvestri, Phys. Rev. B 78, 174422 (2008).

[14] M. Haag, C. Illg, M. Fähnle, Phys. Rev. B 90, 104417 (2014).

[15] M. Krauß, T. Roth, S. Alebrand, D. Steil, M. Cinchetti, M. Aeschlimann, H.C. Schneider, Phys. Rev. B 80, 180407(R) (2009).

[16] B.Y. Mueller, T. Roth, M. Cinchetti, M. Aeschlimann, B. Rethfeld, New J. Phys. 13, 123010 (2011).

[17] U. Atxitia, O. Chubykalo-Fesenko, J. Walowski, A. Mann, M. Münzenberg, Phys. Rev. B 81, 174401 (2010).

[18] T. Roth, A.J. Schellekens, S. Alebrand, O. Schmitt, D. Steil, B. Koopmans, M. Cinchetti, M. Aeschlimann, Phys. Rev. X 2, 021006 (2012).

[19] J. Schilp, T. Kuhn, G. Mahler, Phys. Rev. B 50, 5435 (1994).

[20] M. Haag, C. Illg, M. Fähnle, Phys. Rev. B 87, 214427 (2013).

[21] O.K. Andersen, O. Jepsen, Phys. Rev. Lett. 53, 2571 (1984).

[22] D. Steiauf, C. Illg, M. Fähnle, J. Magn. Magn. Mater. 322, L5 (2010). 